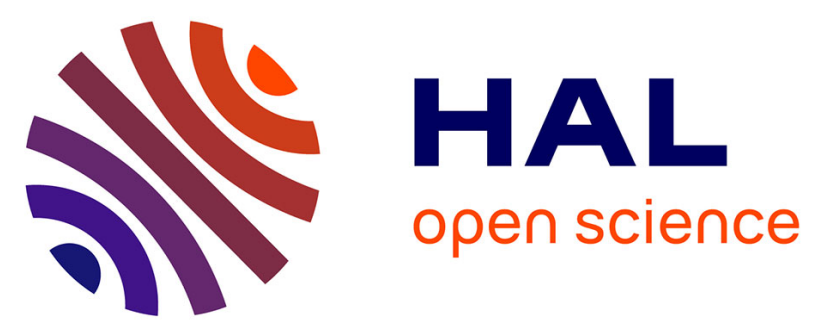

\title{
Ajustement diffus et adaptatif de la consommation électrique résidentielle par un système multi-agent auto-adaptatif
}

\author{
Victor Lequay, Mathieu Lefort, Saber Mansour, Salima Hassas
}

\section{- To cite this version:}

Victor Lequay, Mathieu Lefort, Saber Mansour, Salima Hassas. Ajustement diffus et adaptatif de la consommation électrique résidentielle par un système multi-agent auto-adaptatif. Revue des Sciences et Technologies de l'Information - Série RIA: Revue d'Intelligence Artificielle, 2017, 31 (4/2017), pp.427-447. 10.3166/ria.31.427-448 . hal-01578976

\section{HAL Id: hal-01578976 https://hal.science/hal-01578976}

Submitted on 30 Aug 2017

HAL is a multi-disciplinary open access archive for the deposit and dissemination of scientific research documents, whether they are published or not. The documents may come from teaching and research institutions in France or abroad, or from public or private research centers.
L'archive ouverte pluridisciplinaire HAL, est destinée au dépôt et à la diffusion de documents scientifiques de niveau recherche, publiés ou non, émanant des établissements d'enseignement et de recherche français ou étrangers, des laboratoires publics ou privés. 


\title{
Ajustement diffus et adaptatif de la consommation électrique résidentielle par un système multi-agent auto-adaptatif
}

\author{
Victor Lequay ${ }^{1,2}$, Mathieu Lefort ${ }^{1}$, Saber Mansour ${ }^{2}$, \\ Salima Hassas ${ }^{1}$ \\ 1. Équipe SMA, LIRIS, Université Claude Bernard Lyon 1, Lyon, France \\ [victor.lequay,mathieu.lefort,salima.hassas]@liris.cnrs.fr \\ 2. Ubiant SA, Lyon, France \\ saber.mansour@ubiant.com
}

RÉSUMÉ. L'effacement diffus est un moyen économique et écologique pour garantir l'équilibre du réseau électrique, en alignant la consommation sur la production. La problématique soulevée dans ce contexte consiste à faire en sorte qu'un ensemble de foyers puisse d'une part anticiper sa demande et, d'autre part, ajuster collectivement son effort d'effacement. En choisissant une approche ascendante au problème de l'effacement diffus nous proposons un modèle décentralisé fondé sur un algorithme de diffusion épidémique. Cet algorithme fournit une base robuste et évolutive sur laquelle nous avons construit un mécanisme d'auto-évaluation garantissant la fiabilité nécessaire au fonctionnement d'un réseau électrique, c'est-à-dire la capacité à maintenir un effacement stable. Dans cet article, nous présentons notre système et discutons les résultats que nous avons obtenus sur des données simulées réalistes, puis nous proposons des pistes d'amélioration possibles.

ABSTRACT. Load shedding is a cheap and eco-friendly way to maintain balance on the power grid, by matching consumption on production. In this context, the main problem is to coordinate smart buildings in order for them to be able to anticipate their need and also to collectively adjust their load shedding. We present a bottom-up approach on distributed loadshedding and propose a decentralized model based on gossip protocols. These algorithms provide a strong and scalable platform on top of which we built a self-evaluation mechanism ensuring enough reliability to be used on a large scale power grid, ie the ability to maintain a stable load shedding. In this article, we present our system and discuss the first results we obtained with it, before presenting some of its possible improvements.

MOTS-CLÉS : système multi-agent, ajustement diffus, épidémique, auto-évaluation.

KEYWORDS: multi-agents system, load shedding, gossip algorithm, self-evaluation.

DOI:10.3166/RIA.31.427-448 (c) 2017 Lavoisier

Revue d'intelligence artificielle $-n^{0} 4 / 2017,427-448$ 


\section{Introduction}

Le secteur de l'énergie électrique connaît actuellement de profonds changements. La consommation électrique augmente constamment, proportionnellement au taux d'équipement des foyers. Cette consommation électrique nécessite une augmentation régulière des moyens de production, généralement coûteux et polluants. De plus, ces générateurs sont dimensionnés pour satisfaire la demande lors des pics de consommation, ce qui signifie qu'ils ne fonctionnent pas à pleine capacité le reste du temps, engendrant des coûts supplémentaires. L'effacement de consommation est une solution propre et peu coûteuse à ces problématiques, qui propose de réduire ou de répartir dans le temps la consommation afin de lisser les pics de consommation plutôt que d'augmenter la production (figure 1).

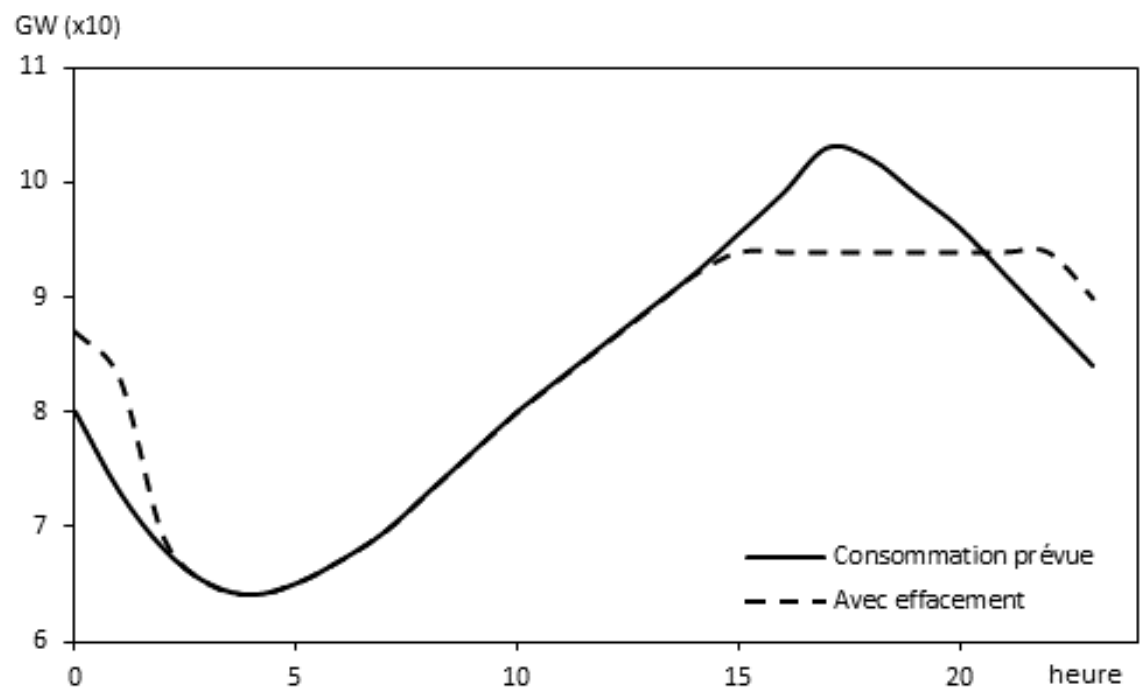

Figure 1. Visualisation de l'effet d'un effacement de consommation.

Données indicatives. Le pic de consommation est effacé par une diminution ou un report de la consommation (ici avant le pic)

Ce procédé existe depuis des années dans le secteur industriel, où les usines sont capables de prévoir avec précision leur consommation plusieurs jours à l'avance et peuvent reporter des consommations importantes à la demande de l'opérateur du réseau. Cependant, ce report est rarement réalisable sur des délais courts et des quantités faibles, car une chaîne de production ne peut pas être arrêtée dans l'heure, ni même du jour au lendemain, et ne présente pas de charges variables. L'application de ce procédé aux plus petits consommateurs, comme les foyers et les commerces, est moins répandue car les problématiques sont multiples. En effet, si le principe est simple, de nombreuses contraintes doivent être satisfaites si l'on veut qu'un tel système s'intègre correctement au réseau électrique et trouve sa place chez le consommateur. Les 
critères de réactivité, stabilité et capacité sont essentiels pour être intégrés au réseau électrique. Si d'une manière générale les systèmes automatisés satisfont largement le critère de réactivité, une incapacité à maintenir un effacement stable et donc le risque d'introduire une nouvelle instabilité sur le réseau annulerait complètement l'intérêt d'un tel système. Il faut donc s'assurer que le mécanisme est capable d'absorber correctement d'éventuelles variations de faible amplitude, et de minimiser le risque d'apparition de variations trop importantes pour être absorbées. La capacité d'effacement, définie par la différence entre la consommation prévue et la consommation réelle pendant l'effacement, est également un critère important, car un système proposant une capacité trop faible pour impacter la stabilité du réseau global n'aurait pas non plus d'intérêt. Du côté de l'utilisateur, le respect du confort et la qualité de service conditionnent l'acceptation d'un tel système par les ménages, sans lesquels l'effacement n'a pas lieu. Par exemple, si le gain financier présenté par l'effacement de consommation est moins important que le désagrément causé par l'extinction des appareils ou le report de leur usage, alors l'habitant risque de ne plus participer au système. Or ce désengagement des participants au système impacte au mieux la capacité potentielle maximale d'effacement disponible si ce désengagement est fait hors effacement (désengagement définitif), au pire sa stabilité si l'utilisateur annule sa participation au cours d'un effacement.

Avec l'approche ascendante décentralisée proposée dans cet article, nous avons donc cherché à résoudre les problèmes suivants. D'une part, maintenir en permanence le niveau de confort de l'occupant pour minimiser le risque de désengagement, et, d'autre part, garantir à chaque instant une stabilité suffisante pour satisfaire les contraintes du réseau électrique.

Nos travaux s'appuient sur Hemis, une solution d'intelligence ambiante pour le foyer développée par la société Ubiant en partenariat avec le laboratoire LIRIS. Hemis fournira au système la capacité d'effacement dont est capable le foyer sans impacter le confort de l'utilisateur. Au-dessus de Hemis, nous proposons une architecture décentralisée permettant l'effacement précis et adaptatif de la consommation d'un grand nombre de foyers. Cette architecture a été conçue avec comme objectif une capacité de passage à l'échelle permettant d'atteindre la centaine de milliers, voire des millions, de foyers connectés sans nécessiter un matériel trop important ou coûteux. L'objectif est donc double : d'une part, permettre une réactivité proche du temps réel pour l'activation d'un effacement de consommation; d'autre part, proposer un système qui soit le plus léger possible tout en étant capable de prendre en charge un maximum d'utilisateurs. Ce sont ces deux contraintes antagonistes qui nous ont amené à choisir une approche décentralisée, que nous développons ici.

Cet article se présente comme suit. En section 2 nous présenterons un état de l'art des travaux liés à la problématique de l'effacement diffus. Nous présentons en section 3 les travaux existants sur lesquels s'appuie ce modèle, puis nous le détaillons dans la section 4 avant de discuter les résultats de nos simulations en section 5 . Nous concluons et discutons les limites et les possibilités d'évolution de notre modèle en section 6 . 


\section{2. État de l'art de l'effacement diffus}

L'effacement de consommation énergétique dans un but d'équilibrage du réseau électrique peut être utilisé sur les points de consommation les plus importants comme les usines par exemple. On parle d'effacement diffus lorsque le principe est appliqué à un grand nombre de consommateurs de plus petite taille, notamment les particuliers. C'est cet aspect qui nous intéressera dans la suite de cet article et le terme effacement renverra, sauf mention contraire, à cette notion. L'effacement est un concept qui englobe de nombreuses problématiques à des niveaux d'abstraction différents. Le système d'effacement doit d'abord être capable de cumuler une capacité d'effacement suffisante pour prétendre participer à l'équilibrage du système. La capacité d'effacement d'un bâtiment résidentiel étant faible par rapport aux quantités d'énergies gérées par le réseau, un nombre important de participants doit pouvoir être géré, d'où la nécessité pour le système de supporter un passage à l'échelle. Cette évolutivité doit également se faire à un coût limité en terme de coûts de communication et de calculs. La réactivité du système est également importante surtout dans l'optique d'une utilisation dans un cadre plus dynamique (ajustement en temps réel (Vedady Moghadam et al., 2014)). La stabilité de l'effacement réalisé est un des défis majeurs des systèmes d'effacement diffus, car un effacement instable n'a aucun intérêt pour le réseau. Enfin, et c'est un point qui n'est presque jamais pris en compte, le respect du confort de l'utilisateur conditionne la satisfaction des contraintes de capacité, de réactivité, et de stabilité.

Le premier point de divergence entre les approches est le type de contrôle utilisé par le système afin de déclencher un effacement. Traditionnellement, l'effacement diffus est réalisé au moyen d'une incitation tarifaire. L'opérateur fait varier le prix de l'énergie pour pousser la consommation des particuliers à la hausse ou à la baisse en fonction de ses besoins de régulation. Cette méthode simple à mettre en place est d'une efficacité relative car la réponse des logements à une variation du prix de l'électricité est difficilement contrôlable donc trop peu prévisible (Callaway, Hiskens, 2011). Elle peut être mal acceptée par le consommateur car si une bonne maîtrise de sa consommation peut lui permettre de réaliser des économies, une erreur de gestion peut avoir un impact très important sur sa facture. Cette approche est donc privilégiée pour les cas d'utilisations dont les contraintes en termes de stabilité et de fiabilité sont les moins grandes, par exemple pour la réduction globale des pics de consommation journaliers. Le prix de l'électricité en tant que signal est néanmoins une base intéressante pour la construction de systèmes distribués (Ramchurn et al., 2011) (Mohsenian-Rad et al., 2010). Plus récemment, l'intérêt des acteurs du domaine s'est porté sur des solutions de gestion plus directes en équipant dans la plupart des cas le foyer du consommateur d'un dispositif permettant de réguler sa consommation de manière plus ou moins automatisée. Cela augmente considérablement le coût du système puisque l'incitation tarifaire ne demande aucun matériel particulier, mais offre plus de réactivité.

La deuxième grande problématique est le moyen de transmission choisi pour échanger des ordres et des informations entre l'opérateur et les équipements installés chez le consommateur. Cela détermine une grande partie du coût du système, sa réactivité et 
son évolutivité. Si un lien direct et permanent est établi entre chaque client et l'opérateur, cela implique une infrastructure réseau importante et coûteuse dans la plupart des cas mais permet une grande réactivité. À l'inverse, si les relais installés dans les foyers ne se connectent qu'à une certaine fréquence, cette fréquence sera critique quant à la capacité du système à répondre aux besoins de l'opérateur. C'est pourquoi la majorité des travaux considèrent une connexion continue aux serveurs de l'opérateur.

Enfin, la problématique traitée ici est le contrôle de ces équipements. Quel algorithme permet de s'engager rapidement et de manière fiable sur une capacité totale d'effacement, puis de la maintenir sur toute la durée demandée tout en respectant le confort des utilisateurs et une certaine équité entre la participation de ces derniers? Les différentes approches existantes dans la littérature peuvent être classées en deux catégories : centralisées quand le contrôle est effectué par un serveur central qui envoie à chaque foyer connecté des ordres simples (coupure/allumage), ou décentralisées quand la décision (de s'effacer ou non) est prise par l'appareil présent dans le foyer. Les algorithmes centralisés proposés dans la littérature et utilisés dans certains cas concrets consistent généralement en un usage plus ou moins poussé de statistiques. Les systèmes historiques, comme la Télécommande Centralisée à Fréquence Musicale d'EDF, permettent l'extinction à distance de certains équipements (le chauffe-eau en général) chez les particuliers ayant souscrit au tarif "heures pleines/heures creuses" d'EDF. Ces extinctions sont généralisées, et leur simultanéité peut avoir un impact important sur le réseau électrique si elles ne sont pas prévues. De même, l'effet de report massif de la consommation dû à l'allumage simultané des équipements après l'effacement peut créer un pic de consommation égal voire parfois supérieur au pic effacé (Callaway, Hiskens, 2011). Cet effet report, amoindri naturellement par les systèmes stochastiques, peut être évité par un système centralisé pour peu que les équipements soient contrôlables individuellement (Lu et al., 2011). Il est alors simple de ne pas réactiver tous les équipements en même temps. Les travaux récents proposant un système centralisé utilisent parfois des techniques tirées du domaine de l'intelligence artificielle comme les réseaux de neurones (Hang et al., 2007) ou l'apprentissage par renforcement (You et al., 2002) afin de déterminer automatiquement les politiques de gestion les plus efficaces.

Dans les approches décentralisées, on trouve les travaux utilisant des systèmes multi-agents. Souvent basés sur une incitation tarifaire (Ramchurn et al., 2011), la plupart proposent un modèle stochastique permettant de répartir l'action des agents dans le temps, évitant ainsi les effets d'oscillations qui peuvent apparaître dans un système basé sur des hystérésis ou des limites de déclenchement trop simples (Vedady Moghadam et al., 2014; You et al., 2002). De même, les travaux de Beal et al. (Ranade, Beal, 2010; Ranade, 2010; Beal et al., 2012; Papalexopoulos et al., 2013) proposent un algorithme nommé ColorPower permettant un contrôle distribué de la demande via un modèle stochastique. Le principe de l'approche stochastique est d'installer chez le consommateur un contrôleur capable de couper l'alimentation de certains appareils. Ce contrôleur éteint ou allume ces appareils avec une probabilité qu'il modifie en permanence, selon un signal envoyé par le serveur central. Ce signal pousse l'augmentation de cette probabilité lors d'un besoin d'effacement et la diminue le reste du 
temps. Ces systèmes ont un inconvénient majeur inhérent à leur forte composante probabiliste : il leur est difficile d'être efficaces avec peu de participants connectés. En effet, d'après ce modèle, la capacité d'effacement disponible n'est pas la somme des capacités disponibles dans chaque foyer équipé car un foyer ne s'efface pas forcément à chaque appel. Les systèmes stochastiques nécessitent donc une quantité de participants minimale pour garantir un effacement de consommation et n'autorisent pas une mise en place itérative du système (Frey et al., 2013).

Le tableau 1 liste les différents critères d'évaluation d'un système d'effacement diffus et permet de situer les différentes approches décrites précédemment.

Tableau 1. Tableau comparatif des différentes approches de l'effacement diffus

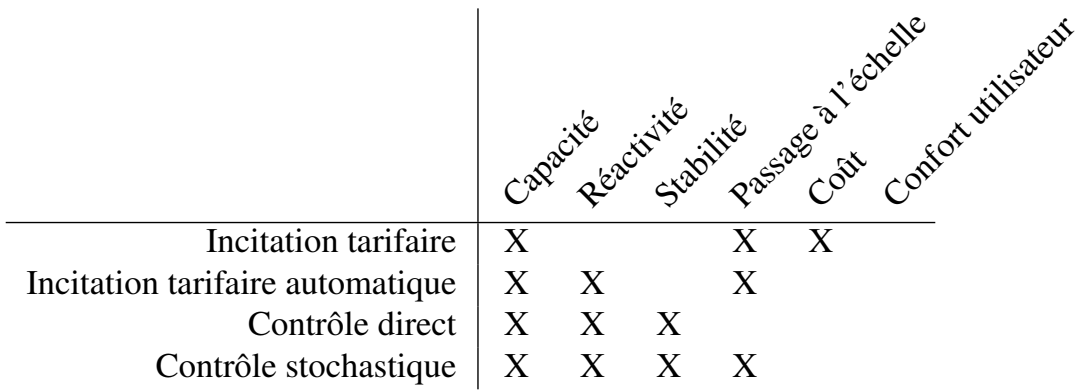

\section{Cadre général}

Dans cette section, nous présentons les systèmes et travaux existants sur lesquels nous nous sommes appuyés pour construire notre système d'effacement. Nous formalisons ensuite le problème que nous cherchons à résoudre.

\subsection{Hemis}

Hemis ${ }^{1}$ est une solution d'intelligence ambiante pour le foyer développée par la société Ubiant en partenariat avec le laboratoire LIRIS. Fondé sur un système d'optimisation multi-contraintes construit sur une logique multi-agents, elle permet de mâ̂triser intelligemment et automatiquement la consommation énergétique du foyer en maintenant le niveau de confort souhaité. D'un point de vue technique, dans Hemis tous les appareils connectés du foyer (chauffage, éclairage, capteurs de présence, de luminosité, volets roulants, etc.) sont représentés par des agents réactifs qui collaborent via des espaces de marquage communs pour atteindre les objectifs de chaque facteur environnemental (température, luminosité, consommation, etc.) et satisfaire leurs contraintes. L'utilisateur fixe simplement les objectifs pour chaque facteur, et les agents agissent de concert pour trouver en temps réel le meilleur compromis entre consommation et confort.

1. http://www.ubiant.com/hemis-ubiant/ 
Pour déterminer la flexibilité disponible, Hemis s'appuie, d'une part, sur un historique local de la consommation et des usages, couplé aux informations données par les capteurs (la courbe de consommation ne sera pas la même que celle des cinq derniers jours s'il fait particulièrement froid le jour même) et, d'autre part, sur les prévisions de chaque agent (capteur ou actionneur) individuellement. La prévision de la flexibilité prendra en compte l'ensemble des équipements consommateurs, qu'ils soient allumés ou non. En effet, l'effacement peut être la coupure d'un équipement mais également le fait de ne pas l'allumer comme d'habitude, en reportant son utilisation ou en l'avançant (chauffer un peu plus tôt une pièce pour profiter de l'inertie thermique pendant l'effacement par exemple). L'utilisateur conserve le contrôle de ses équipements même pendant un effacement. Et si un appareil est allumé alors qu' un effacement est prévu, il n'est pas coupé pour autant. La flexibilité est simplement revue à la baisse. Cela change radicalement le rapport entre l'utilisateur et le mécanisme d'effacement, en opposition totale avec les méthodes existantes avec lesquelles l'occupant subit généralement l'effacement.

Notre système s'appuie sur des solutions capables, comme Hemis, de communiquer en temps réel la flexibilité de chaque foyer qu'elles équipent et d'agir sur sa consommation en temps réel. Il s'agit ensuite d'utiliser ces informations et ces moyens d'actions pour réaliser des effacements stables.

\subsection{Algorithme Push-Sum}

L'algorithme Push-Sum (Kempe et al., 2003) permet à un ensemble d'agents de calculer un agrégat (somme, moyenne, produit, maximum, minimum, etc...) de façon totalement décentralisée en suivant un modèle dit épidémique ou de propagation de rumeur. Dans ce modèle, chaque agent $a$ possède trois variables : $x^{a}$ la valeur participant au calcul de l'agrégat, la somme $s^{a}$ initialisée à $x^{a}$ et la pondération $w^{a}$ initialisée à 0 ou 1 selon le résultat voulu. Dans le cas du calcul d'une somme par exemple, on initialisera $w^{a}$ à 0 pour tous les agents sauf un, choisit au hasard, qui prendra la valeur $w^{a}=1$. À l'instant $t=0$, l'agent s'envoie à lui-même la paire $\left(s^{a}, w^{a}\right)$. À chaque itération, une part $\alpha_{j}^{a}$ de $s^{a}$ et $w^{a}$ est envoyée à un ensemble $J$ d'agents dont lui-même sous la forme $\left\{\left(\alpha_{j}^{a} s^{a}, \alpha_{j}^{a} w^{a}\right) \mid j \in J, a \in J\right\}$ de telle sorte que $\sum_{j \in J} \alpha_{j}^{a}=1$ (classiquement, $\alpha_{j}^{a}=\frac{1}{|J|}$ ).

Les valeurs de $s^{a}$ et $w^{a}$ sont ensuite mises à jour en additionnant les $|K|$ paires $\left\{\left(s^{k}, w^{k}\right)\right\} \mid k \in K$ reçues par $a: s^{a}=\sum_{k \in K} s^{k}$ et $w^{a}=\sum_{k \in K} w^{k}$. À chaque instant, l'agent peut calculer l'estimation de l'agrégat $a g g^{a}=\frac{s^{a}}{w^{a}}$.

Le calcul d'un maximum ou d'un minimum (dans le cas des capacités par exemple, voir 4.3) se fait en retenant toujours le minimum ou le maximum des valeurs reçues pour le propager. Chaque agent envoie alors son propre minimum ou maximum local à ses voisins, sans le pondérer par $\alpha$. Il est intéressant de noter que cet algorithme permet l'implémentation aisée de fonctions d'observation des performances du réseau 
pour un opérateur comme, par exemple, la possibilité de connaître le nombre d'agents possédant certains paramètres (fiabilité, participation, etc.), la moyenne des participations ainsi que d'autres outils statistiques standards (écart type, etc.).

Le choix des voisins lors de l'envoi des messages dépend de la topologie du réseau. Dans le cas d'un graphe complet (chaque agent peut directement communiquer avec n'importe quel autre agent), ce choix peut-être uniformément aléatoire. Il est à noter que cet algorithme ne peut fonctionner que si le réseau peut être représenté par un graphe connexe, c'est-à-dire que pour toute paire d'agents $a$ et $a^{\prime}$ il existe un chemin entre $a$ et $a^{\prime}$.

Kempe et al. montrent que, étant donné un $\gamma>0$ et un $\delta \in[0,1]$ donnés, l'erreur d'estimation de la valeur de l'agrégat $a g g^{a}$ est inférieure à $\gamma$ avec une probabilité supérieure à $1-\delta$ au bout d'au plus $O\left(\log n+\log \frac{1}{\gamma}+\log \frac{1}{\delta}\right)$ itérations. Cette complexité logarithmique autorise un passage à l'échelle rapide.

\subsection{Formalisation du problème}

On suppose par la suite qu'il existe une solution d'intelligence ambiante pour le foyer capable de connaître à tout moment la flexibilité totale $f_{\text {tot }}^{a}(t)$ du logement considéré comme un agent $a$ pour le système, c'est-à-dire la quantité d'énergie effaçable tout en maintenant le confort de l'utilisateur (par exemple la solution Hemis présentée en section 3.1). Cette flexibilité est issue d'estimations et peut varier à tout moment selon les agissements de l'occupant.

Soit un ensemble $A=\left\{a_{1}, a_{2}, \ldots, a_{n}\right\}$ de foyers équipés de cette solution. À chaque instant, on dispose d'une flexibilité totale, i.e une capacité d'effacement totale pour le réseau

$$
F_{\text {tot }}(t)=\sum_{a \in A} f_{\text {tot }}^{a}(t)
$$

Pour satisfaire une demande d'effacement de capacité $\hat{F}_{e}$, chaque agent $a$ participant au système devra engager une part $f_{e}^{a}(t)$ de sa flexibilité. Or, au cours de l'effacement, il est probable qu'un ou plusieurs agents participants voient leur flexibilité diminuer ou même s'annuler, car le système ne contraint pas l'utilisateur (voir section 3.1). Pour garantir la stabilité de l'effacement (c'est à dire minimiser les variations de $F_{e}$ dans le temps), le système doit donc être capable de modifier dynamiquement sa flexibilité $f_{e}^{a}(t)$ pour avoir

$$
\forall t, F_{e}=\sum_{a \in A} f_{e}^{a}(t)=\hat{F}_{e}(t)
$$

Nous proposons dans la section suivante une architecture capable d'assurer cette stabilité en permanence, de façon décentralisée et sans incitation tarifaire. 


\section{Modèle pour l'effacement diffus}

\subsection{Principe}

Afin de répondre au problème exposé en 3.3, nous avons choisi une approche décentralisée fondée sur un système multi-agents utilisant sur un modèle de diffusion épidémique. Un agent est un foyer capable de connaître en temps réel sa flexibilité, par exemple avec la solution domotique décrite en section 3.1. Grâce à un modèle de calcul d'agrégation décentralisé décrit à la section 3.2, chaque agent connaît également en permanence la valeur d'un certain nombre de variables concernant la population totale. Le rôle du système décentralisé est de déterminer dans quelle proportion chaque agent doit participer à chaque ordre d'effacement. Chaque agent possède pour cela une note calculée selon une méthode décrite en section 4.2. Lorsqu'un ordre d'effacement parvient à l'agent, celui-ci va déterminer l'effort qu'il peut et doit fournir en fonction de sa note, de sa flexibilité totale et bien sûr de la demande. Le mécanisme d'engagement est détaillé en section 4.3. Au cours de l'effacement, chaque agent connaît à tout moment une estimation décentralisée de la capacité engagée par la population et ajuste sa propre participation en cas de variation par rapport à l'objectif, comme décrit en section 4.4. L'événement terminé, il mettra à jour sa note en fonction de ses dernières performances.

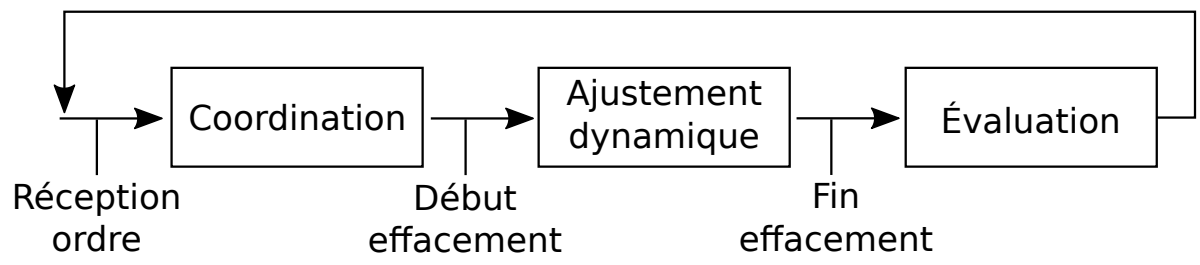

Figure 2. Phases principales de l'exécution d'un agent

\subsection{Auto-évaluation de l'agent}

La note d'un agent évalue sa fiabilité, c'est-à-dire sa capacité à réaliser l'effacement auquel il s'était engagé. En effet, un agent incapable de prédire correctement sa flexibilité impacte négativement l'ensemble du système et signifie que l'occupant du foyer est réticent à participer aux effacements, sa participation aux efforts futurs doit donc être limitée. Pour cela, l'agent calcule à chaque instant l'écart entre son engagement de départ et l'effacement réellement effectué. Afin de sanctionner d'avantage les écarts importants par rapport aux écarts faibles et tardifs, la mesure enregistrée est l'écart quadratique $\left(\hat{f}_{e}^{a}(t)-f_{e}^{a}(t)\right)^{2}$ avec $\hat{f}_{e}^{a}(t)$ l'effacement promis par l'agent à l'instant $t$ pouvant varier en cas d'ajustement de l'engagement (cf. section 4.4) et $f_{e}^{a}(t)$ l'effacement réel de l'agent à l'instant $t$.

Afin de favoriser les agents offrant une grande flexibilité au système, la somme de ces écarts sur la durée de l'effacement $T$ est pondérée par l'inverse de l'engagement au 
carré. Ainsi, plus l'écart représente une part importante de l'engagement, plus l'agent sera pénalisé. On a donc

$$
e^{a}=\sum_{t=1}^{T} \frac{\left(\hat{f}_{e}^{a}(t)-f_{e}^{a}(t)\right)^{2}}{\hat{f}_{e}^{a}(t)^{2}}
$$

Si l'agent ne s'est pas engagé lors du dernier effacement, il retient un écart moyen $e^{a}=0.5$ pour ne pas être pénalisé ni avantagé par un effacement auquel il n'a pas participé.

Afin d'évaluer l'agent non pas sur sa dernière participation mais sur son comportement moyen au fil des effacements récents, la valeur utilisée est une moyenne mobile exponentielle $\bar{e}^{a}$ calculée sur les précédents événements selon la formule $\bar{e}^{a}=$ $(1-\tau) * \bar{e}^{a}+\tau * e^{a}, \tau$ étant la constante de lissage (ou horizon temporel). Cette méthode de calcul de la moyenne évite de devoir conserver des données en mémoire.

Enfin, la note finale $g^{a}$ d'un agent est obtenue après normalisation par les valeurs minimales et maximales de fiabilité obtenues par la population :

$$
g^{a}=\frac{\bar{e}^{a}-e_{\max }}{e_{\min }-e_{\max }} \in[0,1]
$$

Les variables $e_{\min }$ et $e_{\max }$ sont obtenues par l'agent grâce à l'algorithme PushSum (section 3.2).

\subsection{Engagement de la flexibilité}

Un ordre d'effacement $O$ est un ensemble $O=\left\{\hat{F}_{e}, t_{d}, t_{f}\right\}$ comprenant $\hat{F}_{e}$ la quantité d'énergie totale à effacer par le système, supposée constante entre $t_{d}$ la date de début de l'événement et $t_{f}$ la date de fin. Nous considérons ici que l'ordre parvient à un ou plusieurs agents de façon fiable et que chaque agent qui le reçoit le partage à un nombre $j \leq N$ d'agents.

À la réception d'un ordre d'effacement, l'agent détermine son engagement en fonction de sa flexibilité totale courante $f_{\text {tot }}^{a}(t)$ et de sa note de fiabilité $g^{a}$. Un agent ayant une fiabilité maximale s'autorisera à engager toute sa flexibilité. Il conservera tout de même une marge correspondant à une proportion $p \in[0,1]$ de sa flexibilité totale afin de pouvoir augmenter sa participation en cas d'échec d'autres agents (cf section 4.4). Un agent dont la fiabilité est faible s'engagera d'autant moins que sa capacité est grande, afin de moins déstabiliser le système en cas d'échec. En se basant sur les valeurs $f_{\min }$ et $f_{\max }$ (obtenue via Push-Sum decrit à la section 3.2) correspondant respectivement à la capacité totale minimale et maximale engagée par un agent lors du dernier effacement, l'engagement minimum d'un agent (i.e son engagement quand sa fiabilité est nulle) est donné par

$$
f_{\text {base }}^{a}(t)=f_{\min } * \frac{f_{\max }-f_{\text {tot }}^{a}(t)}{f_{\max }-f_{\min }} *(1-p)
$$


Si $f_{\text {tot }}^{a}=f_{\text {min }}$ alors $f_{\text {base }}^{a}=f_{\text {tot }}^{a} *(1-p)$ : un agent avec peu de flexibilité pourra l'engager complètement même si sa note est basse. Si $f_{\text {tot }}^{a}=f_{\max }$ alors $f_{\text {base }}^{a}=0$ : un agent disposant d'une grande capacité ne pourra pas l'engager si sa note est trop mauvaise.

Par interpolation linéaire, l'engagement d'un agent est alors

$$
\hat{f}_{e}^{a}(t)=g^{a} *\left(f_{\text {tot }}^{a}(t) *(1-p)-f_{\text {base }}(t)\right)+f_{\text {base }}(t)
$$

Cet engagement visant à atteindre la capacité demandée $\hat{F}_{e}(t)$, il est borné par l'écart entre $\hat{F}_{e}(t)$ et $F_{e}^{a}(t)$, la capacité totale d'effacement proposée par la population à chaque instant estimée de manière décentralisée grâce à l'algorithme Push-Sum (voir section 3.2) :

$$
\hat{f}_{e}^{a}(t)=\min \left(\hat{f}_{e}^{a}(t),\left(\hat{F}_{e}(t)-F_{e}^{a}(t)\right)\right)
$$

Par ailleurs, les agents recevant tous l'ordre de façon quasi-simultanée (selon la vitesse de diffusion de l'ordre), il est possible que des agents peu fiables s'engagent les premiers, ne laissant qu'une faible part aux agents les plus fiables. Pour éviter cet effet, les agents profitent du temps restant avant le début de l'effacement $\Delta t=\left(t_{d}-t_{r}\right) * \delta$ pour répartir dans le temps leur réaction. $t_{r}$ est ici l'instant de réception de l'ordre et $\delta \in[0,1[$ un ratio permettant de conserver une marge d'ajustement avant le début de l'effacement.

Chaque agent détermine donc le moment où il communiquera son engagement en se basant sur sa fiabilité comme suit :

$$
t_{e}=t_{r}+\Delta t *\left(1-g^{a}\right)
$$

de telle sorte que l'agent le plus fiable s'engage dès réception de l'ordre (à l'instant $t_{r}$ ) et le moins fiable à $t_{r}+\Delta t$.

\subsection{Ajustement de la participation}

Une fois l'engagement terminé et jusqu'à la fin de l'effacement, les agents ajustent leur participation dès que nécessaire pour que l'effacement total $F_{e}(t)$ corresponde à la capacité demandée $\hat{F}_{e}$. Wuhib et al. ont utilisé l'algorithme Push-Sum pour élaborer un mécanisme décentralisé de détection de dépassement de seuil (Wuhib et al., 2010). Nous en avons repris certains éléments et proposons une variante permettant de répartir l'effort de manière décentralisée de façon à ce que l'engagement cumulé des agents converge très rapidement vers l'objectif. Il s'agit dans un premier temps de détecter une différence entre la somme des participations $F_{e}^{a}(t)$ et l'effort demandé $\hat{F}_{e}(t)$. Si comparer les deux valeurs est élémentaire, il faut prendre en compte la variation permanente de la somme des participations dû au temps de convergence de l'algorithme Push-Sum.

Pour éviter un phénomène oscillatoire lorsque $F_{e}^{a}(t) \simeq \hat{F}_{e}(t)$, deux dispositifs ont été introduits. Le premier est un ratio $0 \leq H<1$, implémentant une hystérésis 
autour de l'effort demandé $\hat{F}_{e}(t)$, permettant de ne pas considérer les variations de faible amplitude et de basse fréquence de l'agrégat dues aux changements réguliers de la flexibilité des agents. On considère alors que l'on a un dépassement de seuil si $\left|\frac{F_{e}^{a}(t)}{\hat{F}_{e}(t)}-1\right| \geq H$ (voir figure 3 ).

Le deuxième mécanisme permettant un ajustement fiable est un filtre passe-bas atténuant les variations rapides. Il est implémenté par une durée $t_{\text {wait }}$ pendant laquelle le dépassement doit être constaté avant d'être pris en compte (voir figure 3.

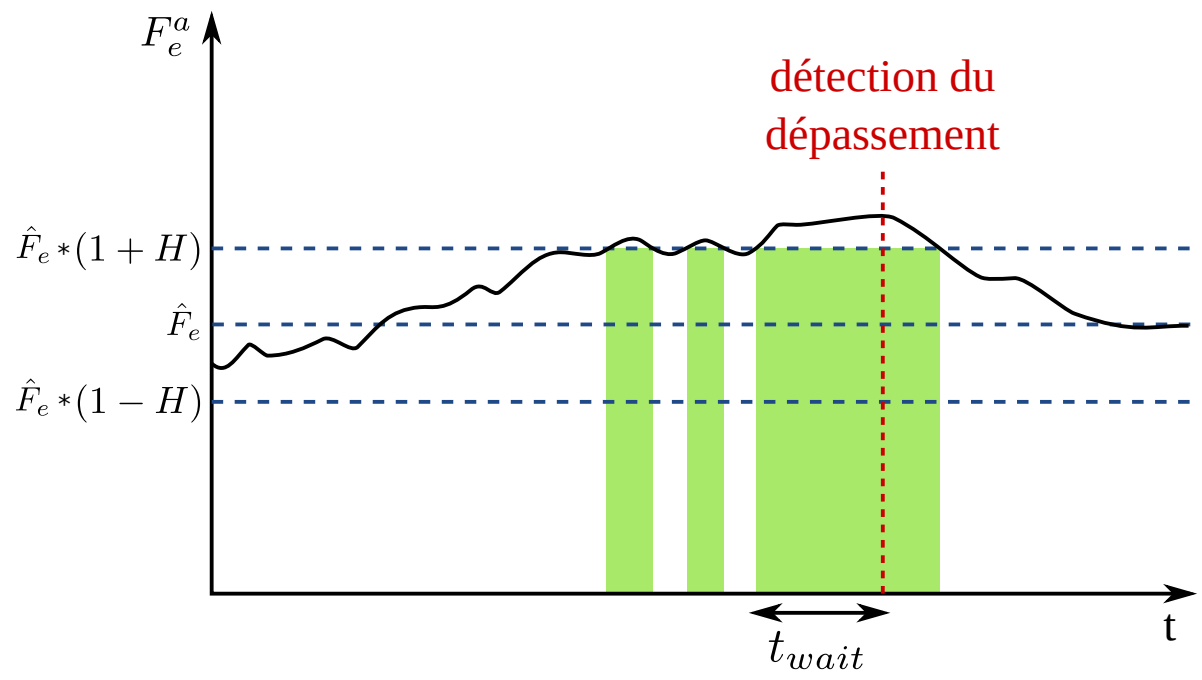

Figure 3. Processus de détection d'un dépassement de l'ordre d'effacement. Le dépassement est avéré lorsque la valeur de l'agrégat sort de l'hystérésis (ici dépasse le seuil $\hat{F}_{e} *(1+H)$ ) (zones grises) et que le délai $t_{\text {wait }}$ est dépassé. L'agent commence alors à ajuster sa participation

Une fois le dépassement détecté, l'agent ajuste sa participation $\hat{f}_{e}^{a}(t)$ suivant la formule

$$
\hat{f}_{e}^{a}(t)=f_{e}^{a}(t)\left(1+\frac{\hat{F}_{e}-F_{e}^{a}(t)}{F_{e}^{a}(t)}\right)
$$

avec $\hat{f}_{e}^{a}(t)$ borné par $f_{\text {tot }}^{a}(t)$. Cela signifie que l'agent augmente sa participation $\hat{f}_{e}^{a}(t)$ de la même proportion que celle nécessaire à $F_{e}^{a}(t)$ pour atteindre $\hat{F}_{e}$. Le délai $t_{\text {wait }}$ doit être défini de telle sorte qu'il permette à la nouvelle valeur de $F_{e}^{a}(t)$ d'être connue par tous avant un nouvel ajustement de la participation, évitant ainsi une oscillation de la valeur de $F_{e}(t)$. 


\section{Expérimentations}

\subsection{Protocole de test}

Pour valider notre modèle, nous simulons un ensemble de $N$ agents dont la flexibilité totale est une valeur en Watt choisie aléatoirement dans l'ensemble $\{100,200,500$, $1000,2000,3000\}$. Les agents disposent donc d'une flexibilité moyenne de 1133W correspondant à la coupure d'un chauffage électrique associée à la diminution légère de la luminosité ambiante. Le taille $N$ de la population a été fixée à 18 pour les résultats présentés ici. Ce nombre peut sembler peut sembler peu significatif comparé aux ordres de grandeurs évoqués dans cet article (plusieurs milliers d'individus au moins), mais il n'est pas arbitraire. En effet, avec un plus grand nombre d'agents les échecs sont moins visibles sur les résultats car ils sont moins significatifs comparés à la capacité totale, alors que le temps de compensation reste le même. Plus le nombre d'agents augmente, plus le système est performant en terme de capacité et de fiabilité. Sa réactivité reste la même. C'est pourquoi nous avons préféré une population plus petite, permettant de réduire le temps d'exécution des simulations et de présenter des résultats plus parlants. L'hystérésis est fixé à $H=0.01$ et le délai du filtre de détection $t_{\text {wait }}$ est de 10 itérations. La constante de lissage du calcul de la moyenne $\tau$ est fixée à 0.1 .

Notre intérêt s'est porté en premier lieu sur la capacité de notre système à réagir aux aléas et à maintenir au mieux un effacement stable, qui est l'objectif principal de notre travail. Nous souhaitions vérifier que, d'une part, notre système est capable de résister à une chute importante de la flexibilité totale (dans la mesure où elle reste au moins égale à la capacité demandée) et que, d'autre part, la qualité de l'effacement est maintenue même lorsque le système doit effectuer des ajustements fréquents (la baisse de flexibilité est répartie dans le temps).

La capacité d'effacement total de notre système dépend uniquement de la capacité qu'a chaque foyer à s'effacer à chaque instant $t$. Toute la flexibilité annoncée par un agent à cet instant est utilisable instantanément. Cependant, elle peut varier par la suite en fonction de l'utilisateur (mise en route ou extinction d'appareils consommateurs par exemple) ou d'éléments extérieurs (météo, etc...). Afin de simuler un comportement crédible des occupants, nous avons donc modulé deux facteurs : la probabilité d'un échec du maintien de la flexibilité engagée, et l'instant où survient cet échec durant l'effacement.

Chaque agent se voit donc attribuer une probabilité d'échec suivant une distribution exponentielle de paramètre $\lambda=\frac{1}{7}$ de manière à avoir un petit nombre d'agents avec une forte probabilité d'échec et inversement. Au cours de l'effacement, si l'agent échoue, sa flexibilité est réduite à zéro, simulant le refus de l'utilisateur à accepter es modifications réalisées par Hemis. Cela représente le pire cas possible mais permet de simplifier le protocole et de mieux éprouver le système tout en facilitant la lecture des résultats, sans pour autant empêcher leur généralisation. 
Le moment où l'agent échouera le cas échéant est ensuite déterminé à chaque effacement selon deux scénario différents selon sa flexibilité totale. Dans le premier cas, si la flexibilité est faible, l'instant $t_{e c h e c}$ est calculé comme suit : $t_{\text {echec }}=t_{d}+\left(t_{f}-\right.$ $\left.t_{d}\right) *(1-\sqrt[3]{\alpha})$ avec $\alpha \in[0,1]$ tiré aléatoirement, $t_{d}$ et $t_{f}$ étant les instants de début et de fin de l'effacement (voir section 3.3). L'agent a donc de grandes chances d'échouer dès le début de l'effacement, puis le risque diminue. Ce premier scénario vise à simuler le cas des foyers présentant une petite capacité d'effacement, qui pourrait représenter une simple réduction de la luminosité ambiante. L'effet de l'effacement étant facilement détectable par l'occupant, celui-ci a de grandes chances de réagir rapidement. Si la flexibilité est plus grande ( $>1000 \mathrm{~W}$ dans notre simulation), $t_{\text {echec }}=t_{d}+\left(t_{f}-t_{d}\right) * \sqrt[3]{\alpha}$ ce qui correspond à une courbe symétrique au premier scénario. Plus le temps passe, plus l'agent risque d'échouer. Ce scénario simule le cas de foyers présentant une flexibilité plus importante qui correspondrait à la coupure d'un ou plusieurs appareils de chauffage, ou au report de l'utilisation d'un appareil électroménager. Dans le cas du chauffage, l'effet de la coupure ne se fait pas sentir immédiatement (inertie thermique) et les occupants seront de plus en plus nombreux à réagir au fur et à mesure que la température diminue.

Pour des raisons de temps de simulation, la durée des effacements et l'intervalle qui les séparent dans nos simulations est réduit à 200 d'itérations chacun, ce qui représente un temps extrêmement court (moins d'une seconde) comparé à la durée d'un effacement de consommation en conditions réelles (plusieurs minutes voire plusieurs heures, avec un préavis d'au moins une demi-heure). Ces temps très courts sont néanmoins suffisant pour que le système fonctionne correctement et que nous puissions mesurer convenablement l'efficacité des différents éléments du modèle.

\subsection{Résultats}

\subsubsection{Ajustement en temps réel}

Afin de tester l'efficacité du système multi-agents pour le maintien de la stabilité de l'effacement, nous avons analysé les performances de notre modèle en désactivant le processus de notation. À la réception de l'ordre, chaque agent s'engage du maximum possible sans tenir compte de sa fiabilité. Le reste du modèle (ajustement en temps réel) fonctionne comme décrit en section 4.4.

La figure 4 montre 18 agents réalisant 2 effacements à la suite (le comportement est similaire à chaque effacement, pour des raisons de lisibilité nous n'en montrons que deux).

La courbe continue représente la flexibilité totale engagée $F_{e}(t)$. À l'origine, la flexibilité est à 0 . À la réception de l'ordre $(\mathrm{t}=30)$, la phase de coordination commence. On observe un pic de l'engagement atteignant la flexibilité totale lorsque les agents s'engagent simultanément de toute leur capacité (pour rappel, l'évaluation est désactivée) puis un ajustement à la valeur demandée $\hat{F}_{e}=10000 \mathrm{~W}$ après une durée correspondant au filtre de détection $t_{\text {wait }}$, ce qui valide le bon fonctionnement de la phase de coordination. Lorsque l'effacement débute $(\mathrm{t}=230)$, on constate la di- 


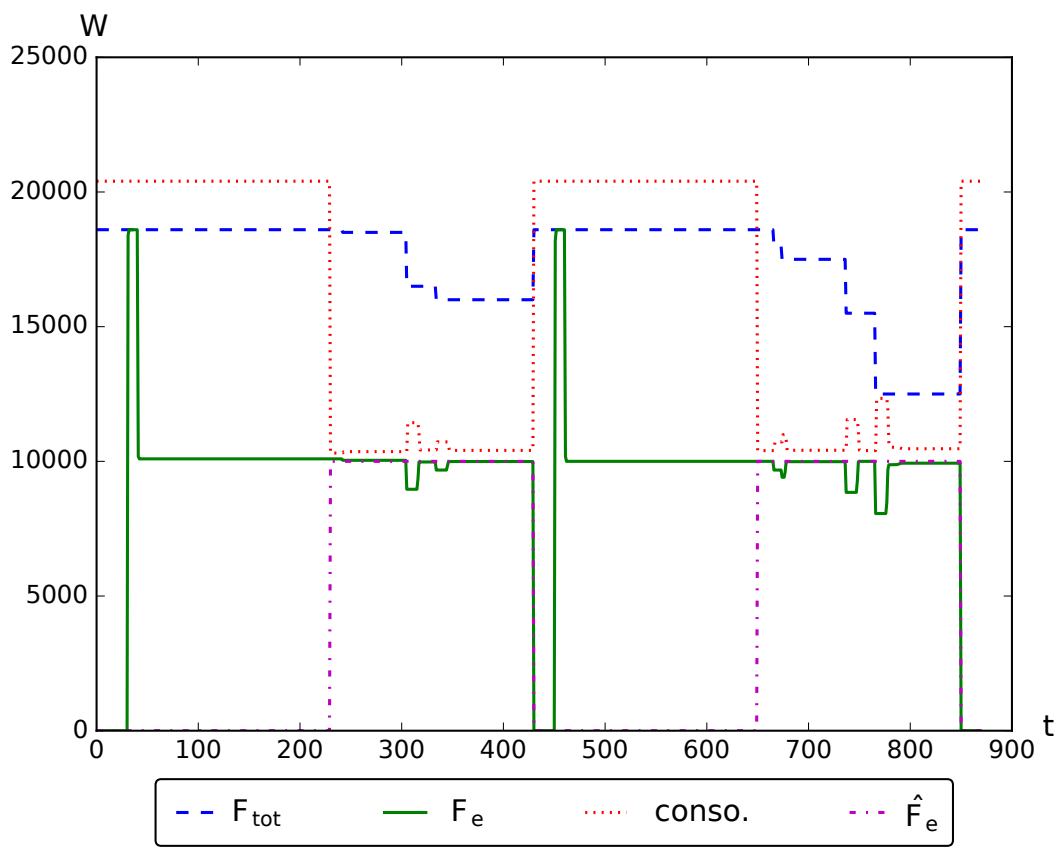

Figure 4. Ajustement dynamique sur deux événements consécutifs

minution de la consommation globale (en pointillés) de la quantité correspondante : l'effacement diffus fonctionne. Au cours de l'effacement, la flexibilité totale disponible $\sum f_{t}$ chute graduellement conformément aux scénarios décrit en section 5.1, montrant l'échec de certains agents. Comme prévu, cette chute est visible sur la flexibilité engagée $\sum f_{e}$, mais est compensée rapidement par d'autres agents au bout d'un temps correspondant au minimum à $t_{\text {wait }}$. Ce délai est le temps minimum de détection d'une variation, comme décrit en section 4.4, et permet de lisser les fluctuations dues à la propagation épidémique des informations. Cette adaptation dynamique à l'échec sans effet de surcompensation, observable lors de chaque variation de l'engagement pendant chaque effacement, confirme l'efficacité du système d'ajustement dynamique.

\subsubsection{Auto-évaluation}

La notation a un impact sur deux éléments distincts à savoir la flexibilité engagée et le temps de réaction de l'agent. Nous avons analysé l'impact de ces deux éléments séparément. Pour évaluer l'efficacité du processus d'évaluation, nous avons mesuré à chaque itération la différence entre la capacité demandée et la capacité effectivement effacée par le système. Si l'évaluation fonctionne, la flexibilité engagée par les agents les moins fiables devraient diminuer au fil des effacements et donc moins impacter a qualité de ces derniers. La figure 5 montre l'évolution de ce critère sur six effa- 


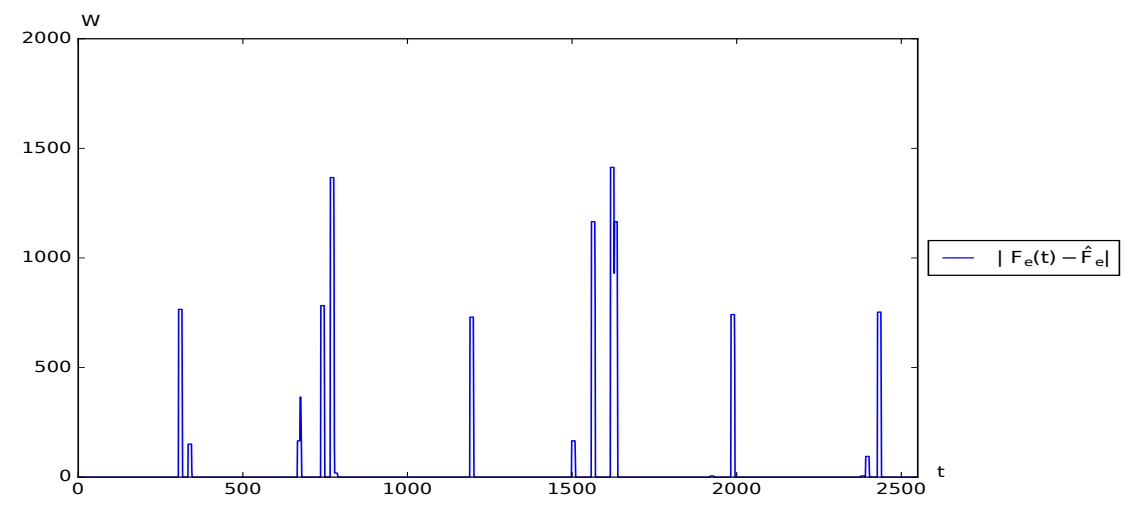

a

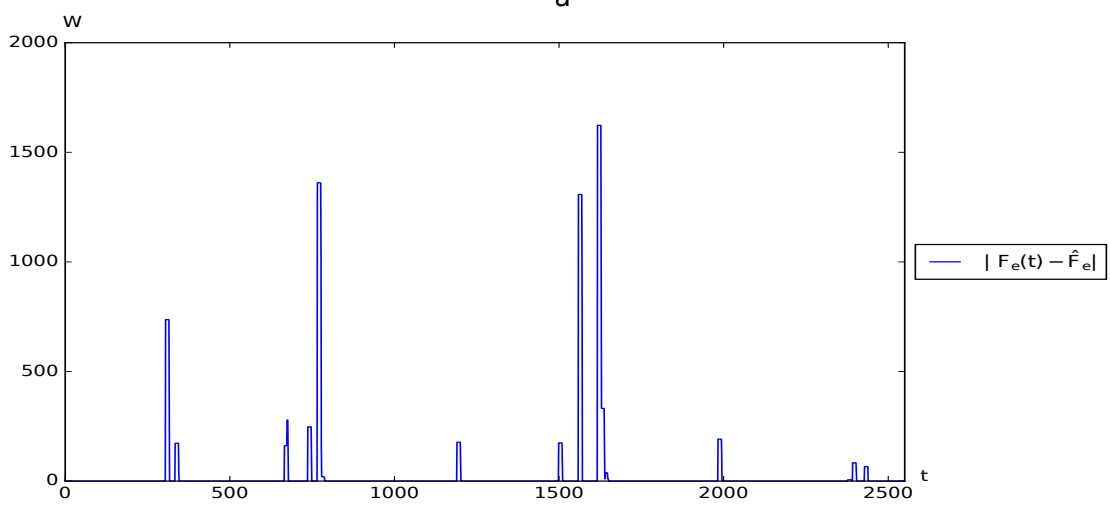

b

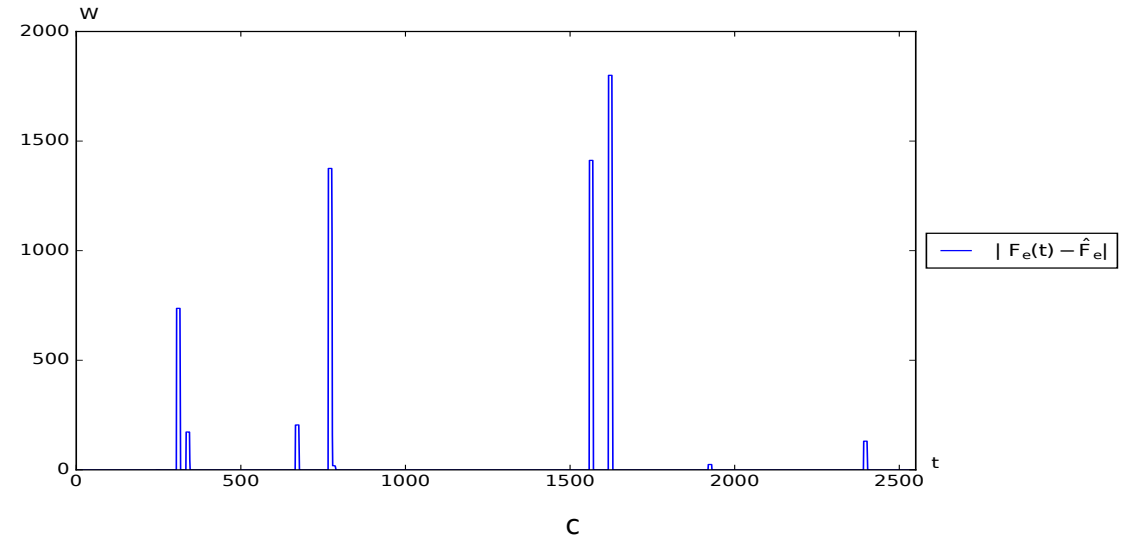

Figure 5. Évolution de la performance sur 6 effacements consécutifs. En (a) les agents s'engagent au maximum dès réception de l'ordre d'effacement. En (b) ils s'engagent dès réception de l'ordre, en fonction de leur fiabilité. En (c), ils s'engagent de la même manière, après un temps déterminé par leur fiabilité 
cements consécutifs. Nous avons conservé les mêmes probabilités d'échecs (random seed identique) au même moment pour permettre une comparaison équitable des modèles. Les résultats montrés sont représentatifs de l'ensemble des simulations menées. Dans un premier temps nous avons mesuré l'amélioration des performances du système lorsque les agents engagent une part de leur flexibilité totale proportionnelle à leur note (voir section 4.2). Cet engagement est effectué dès la réception de l'ordre. Sur la figure 5b, on observe l'évolution de la quantité d'échec à chaque effacement. Pour permettre la comparaison, la figure 5a montre la même mesure dans des conditions identiques, sans le processus d'évaluation. On voit clairement que lorsque les agents pondèrent leur engagement en fonction de leur fiabilité, les performances s'améliorent nettement. Le nombre et la hauteur des pics représentant les défauts d'effacement sont grandement diminués.

\subsubsection{Engagement des agents}

Dans un second temps nous avons mesuré l'impact de la pondération du temps de réaction par la fiabilité. Cela devrait permettre de réduire la part d'aléatoire inhérente au modèle décentralisé et donc augmenter l'impact de la fiabilité sur l'engagement des agents. Sur la figure 5c, on observe bien une diminution de la quantité d'échec encore plus marquée que sur la figure $5 \mathrm{~b}$. Pendant le $5^{e}$ et $6^{e}$ effacement, la capacité demandée est maintenue totalement malgré la chute de la flexibilité totale, seul les agents les plus fiables participant à l'effacement. Cela montre l'efficacité des deux méthodes combinées pour l'amélioration des performances du système à mesure que l'auto-évaluation progresse.

Nous avons ensuite évalué l'intérêt pour les agents de conserver une marge $p$ de flexibilité lors de la phase d'engagement (voir section 4.3). Cela permet de constituer une réserve permettant de compenser le potentiel échec d'autres agents au cours de l'effacement. Pour montrer cet avantage, nous avons effectué la même simulation que précédemment (figure 5) mais cette fois en forçant artificiellement les agents les plus fiables $\left(g^{a}>0.99\right)$ à annuler systématiquement leur flexibilité à partir du quatrième effacement $(\mathrm{t}=1600)$. Les résultats sont visibles sur la figure 6 . Avec $p=0.5$, les agents n'engagent que la moitié de la flexibilité disponible lors de la phase de coordination. L'effort d'effacement est alors réparti sur une large portion de la population car l'objectif n'est pas atteint immédiatement par les premiers agents à s'engager, à savoir les plus fiables. Lorsque ces derniers échouent, représentant tout de même une part conséquente de la flexibilité engagée, l'ajustement se fait sans problème majeur. On remarque bien sûr un échec total plus important. Lorsque $p=1$ (figure 6a), les agents engagent leur flexibilité totale lors de la phase de coordination. L'effort est donc réparti sur un petit nombre d'agents, là encore les plus fiables. Lorsque ceux-ci échouent, l'ajustement est difficile car de nombreux agents ne se sont pas engagés et mettent plus de temps à ajuster leur participation. Cela montre que le paramètre $p$, en permettant de mieux répartir l'effort sur la population d'agent, augmente la résilience du système. 

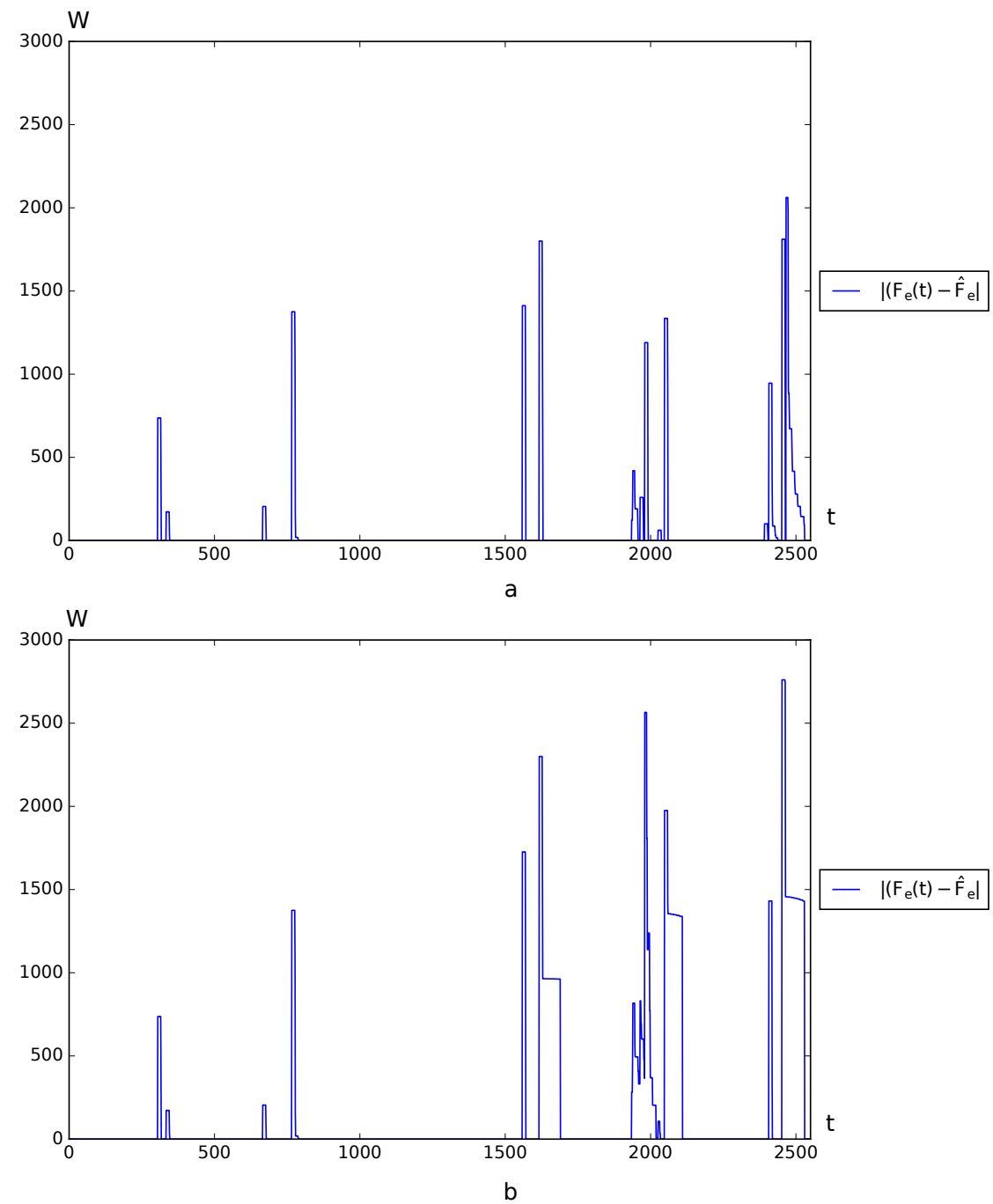

Figure 6. Évolution de la performance sur 6 effacements consécutifs. En (a) les agents conservent une part de leur flexibilité en réserve pour pallier d'éventuels échecs futures. En (b) ils s'engagent de toute la flexibilité possible en fonction de leur fiabilité 


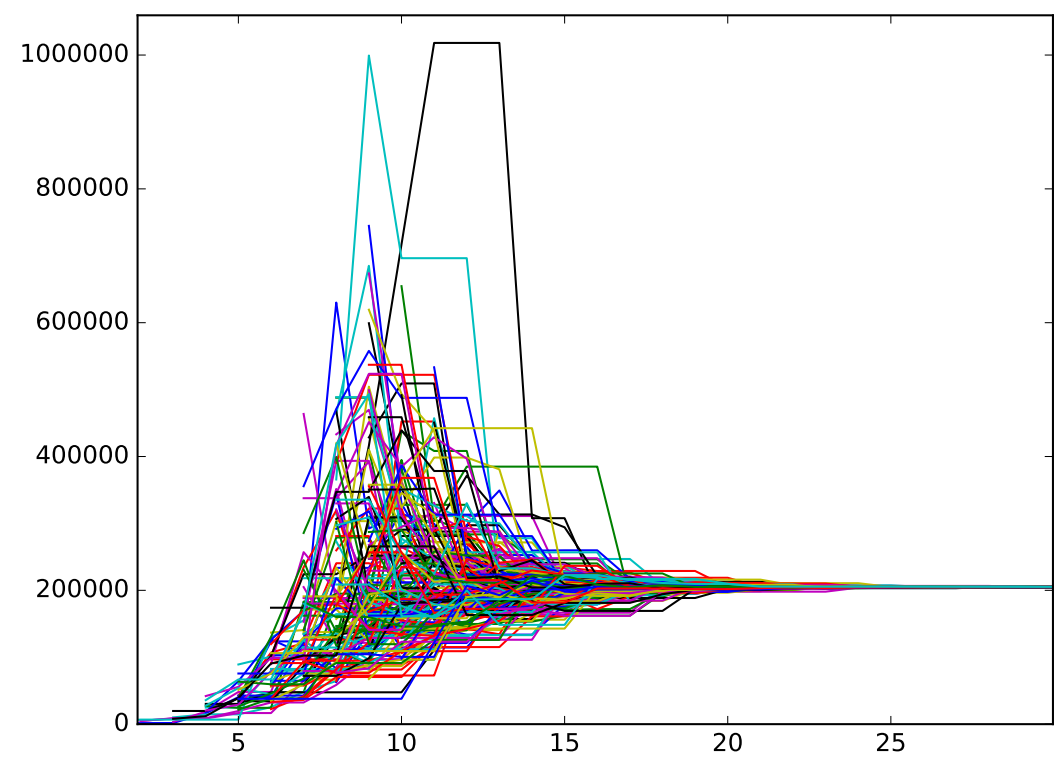

Figure 7. Convergence de la valeur de l'agrégat calculée de manière décentralisée grâce à l'algorithme Push-Sum. 200 agents communiquant à chaque itération avec un seul voisin tiré au hasard dans la population

\subsubsection{Communication décentralisée}

Enfin, nous avons fait varier le nombre de voisin avec lesquels communique chaque agent pour le calcul des agrégats, afin de s'assurer que notre système fonctionne même sous de forte contraintes de connectivité. Nous avons donc simulé un ensemble de 200 agents ne communiquant chacun qu'avec un seul voisin. La figure 7 montre pour chaque agent la valeur de l'agrégat représentant la participation totale de la population au lancement du système. Initialisée pour chaque agent à leur flexibilité totale, elle converge en 20 itérations vers la valeur exacte (210 000). Cela montre que malgré un nombre important de participants et une connectivité minimale, la valeur des variables partagées est connue par l'ensemble de la population en une vingtaine d'itération (ce qui correspond avec notre implémentation à moins d'une demi-seconde). Cela confirme la pertinence d'un tel mécanisme pour une utilisation en situation réelle, et ouvre même la voie à une utilisation dans le cadre d'un ajustement permanent en temps réel de la consommation. 


\section{Conclusion et perspectives}

L'effacement diffus est une technique vouée à se développer rapidement dans les prochaines années. Solution logique aux problématiques actuelles sans obstacle technique majeur, elle offre aux énergéticiens un levier écologique pour maintenir la stabilité du réseau et aux consommateurs un moyen simple et intelligent de réaliser des économies d'énergie. L'obstacle majeur des systèmes actuellement en place et des mécanismes développés dans la littérature est de concilier le respect du confort utilisateur, avec le maintien d'une complexité acceptable. Or, prendre en compte l'utilisateur implique pour un modèle centralisé un échange à double sens permanent entre un nœud central et ses utilisateurs, et donc une montée en charge difficile. Pour pallier ces problèmes, nous proposons une architecture décentralisée pour l'effacement diffus, capable de supporter la gestion d'un grand nombre de consommateurs simultanément, conçue en suivant un raisonnement ascendant. En déléguant le maintien du confort utilisateur au foyer lui-même et en laissant les agents décider indépendamment de leur participation à un effort d'effacement, on augmente drastiquement la résilience du système, ce qui autorise alors une grande évolutivité sans nécessiter de ressources plus importantes, au contraire des méthodes stochastiques où un effacement efficace n'est possible qu'à partir d'un certain nombre de participants. La flexibilité déclarée par les agents est mobilisable instantanément à tout moment, permettant une mise en place itérative du système qui est utilisable dès le premier utilisateur connecté.

Nous avons proposé dans cet article un système de coordination et d'auto-évaluation des agents utilisant un algorithme épidémique permettant à chaque individu de connaître à tout moment différentes informations concernant la population entière, sans nécessiter de nœud central ou de connectivité trop importante. Afin de nous assurer de la pertinence de cet algorithme de propagation pour une utilisation en situation réelle, nous avons vérifié en simulation que le temps nécessaire à l'obtention par chaque agent de la valeur correcte d'une variable partagée était suffisamment court pour permettre une bonne réactivité, et ce même lorsque la population atteint une taille importante. La plus importante de ces variables est la capacité d'effacement totale engagée à chaque instant par l'ensemble de la population. Elle permet à chaque agent d'ajuster sa propre participation pour maintenir la stabilité de l'effacement. Pour que cet ajustement soit efficace, un mécanisme d'hystérésis combiné à un filtre passe-bas est utilisé pour ne pas considérer les variations dues à la propagation décentralisée et éviter des phénomènes oscillatoires.

Sur cette base de communication fiable, nous avons construit un système d'autoévaluation permettant de favoriser la participation des agents les plus fiables à l'effort d'effacement diffus. Pour cela, chaque agent mesure lors d'un effacement son erreur quadratique par rapport à son engagement initial. Si la flexibilité qu'il fournit réellement au système diverge trop de celle qu'il avait annoncé, sa note diminuera. La capacité qu'il engagera lors du prochain effacement sera alors plus faible, afin de moins impacter la stabilité du système s'il échoue à nouveau. Cette incapacité potentielle à maintenir l'effacement auquel l'agent s'était engagé provient de notre volonté de ne pas impacter négativement le confort de l'occupant, qui se manifeste notamment 
par la libre utilisation des appareils électriques à n'importe quel moment. Si le mécanisme d'ajustement dynamique permet de compenser ces aléas, l'auto-évaluation des agents permet d'apprendre dans la durée le comportement de l'occupant pour améliorer la résilience du système sur le long terme. Pour valider notre approche, nous avons mesuré les performances de notre système sur des simulations que nous avons voulues réalistes, notamment concernant l'échec de l'effacement des agents. Afin de simuler au mieux le comportement des occupants, nous avons fait varier à la fois la probabilité de l'échec d'un agent pendant un effacement, ainsi que le moment auquel surviendrait cet échec au cours de l'effacement. Nous avons d'abord confirmé grâce à nos simulations l'efficacité de l'ajustement dynamique. Chaque écart avec l'objectif est compensé dès qu'il est détecté. Nous avons ensuite montré l'intérêt du processus d'auto-évaluation en mesurant l'évolution du nombre d'échecs au fil des effacements. L'auto-évaluation réduisant l'impact des agents les moins fiables sur la qualité de l'effacement, cette dernière s'accroît avec la diminution du nombre d'échecs. En comparaison, le nombre d'échecs sans auto-évaluation reste constant. Ces simulations mettent également en évidence le besoin d'un étalonnage préalable à la réalisation d'effacements réels, l'ajustement de l'auto-évaluation étant le résultat d'un apprentissage sur plusieurs effacements.

Ces résultats prometteurs amènent à un certain nombre de perspectives. Dans un premier temps, nous devrons soumettre notre système à des tests plus poussés en conditions réelles, plus contraignants en termes de résistance aux pannes, de topologie du réseau et de connectivité. Cela nous permettra de mieux quantifier les limites de notre système selon l'usage visé.

Il nous semble également intéressant d'intégrer un processus d'optimisation des différents paramètres locaux, tels que l'hystérésis $H$ ou le délai $t_{\text {wait }}$, ainsi que la constante de lissage $\tau$ et la marge d'engagement $p$. Ces paramètres pourraient être modifiés par l'agent en fonction d'observations locales (détections de dépassement trop fréquentes ou peu pertinentes, participation trop faible malgré une grande fiabilité) puis les résultats propagés sur le réseau en fonction de leur résultat.

Dans un deuxième temps, il serait intéressant d'améliorer la façon dont les agents reçoivent l'ordre d'effacement. En effet, en l'état actuel, l'ordre d'effacement est communiqué à un ou plusieurs agents en provenance d'une source extérieure unique et considérée comme fiable. Cela rend cependant le système dépendant de cette source (l'opérateur du réseau électrique en l'occurrence). Nous pensons qu'un mécanisme d'apprentissage couplé à un réseau de confiance permettrait aux agents de prévoir les besoins en termes d'effacement. Cela permettrait à terme de se passer de source d'information extérieure unique et d'anticiper au plus tôt l'arrivée d'un ordre d'effacement, augmentant la résilience du système.

\section{Bibliographie}

Beal J., Berliner J., Hunter K. (2012). Fast Precise Distributed Control for Energy Demand Management. In Self-Adaptive and Self-Organizing Systems (SASO), 2012 IEEE Sixth In- 
ternational Conference, p. 187-192. IEEE. (00000)

Callaway D. S., Hiskens I. A. (2011). Achieving Controllability of Electric Loads. Proceedings of the IEEE, vol. 99, no 1, p. 184-199. (00000)

Frey S., Diaconescu A., Menga D., Demeure I. (2013). A Holonic Control Architecture for a Heterogeneous Multi-Objective Smart Micro-Grid. In, p. 21-30. IEEE. (00000)

Hang J., Tian J.-X., Lin H.-G. (2007). Application of artificial neural network in intelligent building. In 2007 International Conference on Machine Learning and Cybernetics, vol. 7, p. 4215-4220. IEEE. (00006)

Kempe D., Dobra A., Gehrke J. (2003). Gossip-based computation of aggregate information. In Proceedings. 44th Annual IEEE Symposium on Foundations of Computer Science, 2003, p. 482-491. IEEE.

Lu S., Samaan N., Diao R., Elizondo M., Jin C., Mayhorn E. et al. (2011). Centralized and decentralized control for demand response. In Innovative Smart Grid Technologies (ISGT), 2011 IEEE PES, p. 1-8. IEEE. (00070)

Mohsenian-Rad A.-H., Wong V. W. S., Jatskevich J., Schober R., Leon-Garcia A. (2010). Autonomous Demand-Side Management Based on Game-Theoretic Energy Consumption Scheduling for the Future Smart Grid. IEEE Transactions on Smart Grid, vol. 1, n ${ }^{\circ} 3$, p. 320-331. (00000)

Papalexopoulos A., Beal J., Florek S. (2013). Precise Mass-Market Energy Demand Management Through Stochastic Distributed Computing. IEEE Transactions on Smart Grid, vol. 4, $\mathrm{n}^{\circ}$ 4, p. 2017-2027. (00000)

Ramchurn S. D., Vytelingum P., Rogers A., Jennings N. (2011). Agent-based control for decentralised demand side management in the smart grid. In The 10th International Conference on Autonomous Agents and Multiagent Systems-Volume 1, p. 5-12. International Foundation for Autonomous Agents and Multiagent Systems. (00000)

Ranade V. V. (2010). Model and control for cooperative energy management. Raytheon BBN Technologies. (00000)

Ranade V. V., Beal J. (2010). Distributed control for small customer energy demand management. In 2010 Fourth IEEE International Conference on Self-Adaptive and Self-Organizing Systems, p. 11-20. IEEE. (00000)

Vedady Moghadam M. R., Ma R. T. B., Zhang R. (2014). Distributed Frequency Control in Smart Grids via Randomized Demand Response. IEEE Transactions on Smart Grid, vol. 5, no 6, p. 2798-2809. (00000)

Wuhib F., Dam M., Stadler R. (2010). A gossiping protocol for detecting global threshold crossings. Network and Service Management, IEEE Transactions, vol. 7, n ${ }^{\circ}$ 1, p. 42-57. (00000)

You H., Vittal V., Jung J., Liu C.-C., Amin M., Adapa R. (2002). An intelligent adaptive load shedding scheme. Proc. 2002 14-th PSCC, p. 17-6. (00013) 